\title{
Management Data Specification for Supply Chain Integration
}

\section{Y. T. Lee}

U. S. DEPARTMENT OF COMMERCE Technology Administration National Institute of Standards and Technology

Gaithersburg, MD 20899

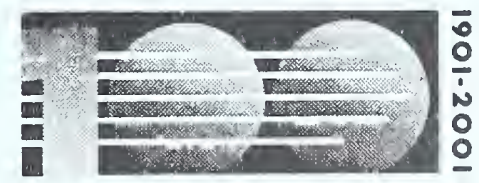

NIST CENTENNIALE

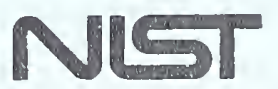

Mational Instifute of Standards and Technology

Technology Administration

U.S. Department of Commerce 



\section{Management Data Specification for Supply Chain Integration}

Y. T. Lee

U.S. DEPARTMENT OF COMMERCE Technology Administration National Institute of Standards and Technology

Gaithersburg, MD 20899

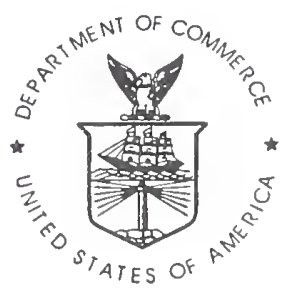

U.S. DEPARTMENT OF COMMERCE Donald L. Evans, Secretary

NATIONAL INSTITUTE OF STANDARDS AND TECHNOLOGY

Dr. Karen H. Brown, Acting Director 



\title{
Management Data Specification for Supply Chain Integration
}

\author{
Shigeki Umeda ${ }^{1}$ \\ Management School \\ Musashi University \\ 1-26-1 Toyotama-kani \\ Nerima-ku Tokyo 176-8534 \\ Japan \\ Y. Tina Lee \\ Manufacturing Systems Integration Division \\ National Institute of Standards and Technology \\ Gaithersburg, MD 20899-8260 \\ USA
}

\begin{abstract}
This document specifies a framework of communication data in a supply chain system, which is viewed as a virtual enterprise. The framework is developed to support the Intelligent Manufacturing

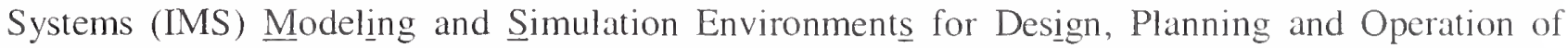
Globally Distributed Enterprises (MISSION) project. A requirement analysis is presented to identify the information necessary to represent the communication for the production management of a supply chain. The requirement analysis has been based on the practical business operations in a U.S. manufacturing company. A specification that describes all information requirements using the eXtensible Markup Language (XML) is presented. The specification will be used to synchronize the business operations within a supply chain simulation system. The specification can also be the basis for developing applications to support the supply chain integration. Such applications can be developed using a database management system or a knowledge base system.
\end{abstract}

\section{KEYWORDS}

Data exchange, information modeling, supply chain, system integration, XML

Certain commercial software and hardware products are identified in this paper. This does not imply approval or endorsement by NIST, nor does it imply that the identified products are necessarily the best available for the purpose.

\subsection{Introduction}

The Manufacturing System Integration Division (MSID) of the National Institute of Standards and Technology (NIST) is participating in the Intelligent Manufacturing Systems (IMS) Modeling and Simulation Environments for Design, Planning and Operation of Globally Distributed Enterprises (MISSION) project. IMS is an industry-led, international research and development program established to develop the next generation of manufacturing and processing technologies. MISSION is an international collaborative project within the IMS Program. "The goal of MISSION is to integrate and utilise new, knowledge-aware technologies of distributed persistent data

\footnotetext{
${ }^{1}$ Research performed while the author is a guest researcher at the National Institute of Standard and Technology.
} 
management, as well as conventional methods and tools, in various enterprise domains, to meet the needs of globally distributed enterprise modeling and simulation [1]." Currently, there are three MISSION project teams: the United States team, the Europe team, and the Japan team.

A distributed manufacturing simulation architecture [2] has been developed by the NIST MSID to support the MISSION project. The architecture describes the major system modules, data elements or objects, and interfaces between modules. The goal of the architecture is to identify the software building blocks and interfaces that will facilitate the integration of a distributed simulation system and enable the integration of those systems with other manufacturing software applications. The architecture, however, does not address the detailed design of individual modules, the information models for shared data elements or objects. To develop an information model and to build a prototype system are the current research emphasis at MSID.

A supply chain simulation prototype system (or in short, supply chain simulation) is being developed as a test case for the MISSION project by the U.S. project team. In an early planning meeting by the U.S. team, a common interest in the supply chain simulation was established. The objective of MISSION is focused on the globally distributed virtual enterprise. In a globally distributed virtual enterprise, supply chain issues are important. The test case focuses on modules, data structures, and interfaces that require an information model.

The supply chain simulation is intended to support the following environment:

- It uses a hybrid push-pull distribution system for product distribution.

- It uses a mature communication mechanism among chain members.

- It uses a centralized control distribution system for communication.

- It may use third-party service providers.

- It may include both simulation and non-simulation software applications.

- It may include multiple software applications in different locations.

- It may include multiple software applications that are executing independently and interacting with each other.

- It may comprise multiple functional modules, such as transportation networks, simulation engines, display systems, etc.

- It spans across multiple businesses and organizations.

- It may simulate multiple levels of manufacturing systems.

- It may allow multiple simulations with different simulation requirements.

Supply chain management is a set of approaches utilized to integrate suppliers, manufacturers, warehouses, and stores efficiently, so that a merchandise is produced and distributed at the right quantities, to the right locations, and at the right time, to minimize system-wide costs while satisfying service level requirements [3]. In a supply chain system, an individual chain member exchanges data with other members to synchronize their business operations. These exchanged data generally include product specification data, planning data, ordering data, inventory data, etc. These data are often used to control operations in an individual firm, and are also used for negotiation among chain members that form a virtual organization to provide products and services to customers. 
The manufacturing management data can be classified into three categories: (1) Order data, (2) Plan data, and (3) Monitor data. "Order" is to assign resources to the operational activities such as manufacturing, transportation, storing, etc. "Plan" is to define a set of "Orders" in the preproduction phase. "Monitor" is to observe/manage the operational activities. These data are generated, used, and discarded according to the individual process activities.

Building a well-organized supply chain requires a well-defined communication mechanism to provide a precise and complete set of definitions for both business communication and transaction data. A communication mechanism will be needed to manage individual chain member companies. It can also be useful for creating an operation strategy in a supply chain. Furthermore, the data specification for the chain can be used to support the design of a distributed database among companies using Electronic Data Interchange (EDI) [4] or an internet protocol.

There are several information modeling methodologies, modeling languages, and implementation methods available to support the development of such communication mechanisms [5]. Our approach to developing this communication mechanism and the data specification are listed here:

- Perform a case study to investigate a real supply chain system.

- Identify the scope of the target application.

- Identify core processes of supply chain management.

- Analyze communication data flow.

- Layout a data specification.

This paper provides a generic specification of supply chain management data, with which the chain members use to communicate with one another. The paper first describes a configuration of a supply chain system. It then details the data requirements to integrate operations in the supply chain system. Finally, the paper presents a data specification for the data requirements using the eXtensible Markup Language (XML).

\subsection{Scenario and Scope}

In this section, a case study of a supply chain company group that manufactures power tools is described. This supply chain company group is composed of several parts suppliers, product warehouses, distributors, retailers, and transportation providers. The organizations of the supply chain include a headquarters, a final assembly plant, parts suppliers, and warehouses. The final assembly plant manufactures products by using the parts provided from the parts suppliers. The warehouses store the distribution inventories and supply the products to the customers. The headquarters of this contract company plays an important role in managing information flows in this chain and also provides the products to the customers through the retailers.

The headquarters, a final assembly plant, and the warehouses belong to the same company. They can share management information at any time by using a common management database. The other chain members are basically organizations of the other companies, which are independent of each other. They are business partners to provide common products to customers; however, they have independent management policies and they pose different management strategies to make their own profit. This is a typical example of a supply chain system of discrete manufacturing. 
In the case study, the supply chain poses two characteristics in the view of production operations management. The first is that this supply chain system owns two streams of product flows, which depend on the product type. The second is that this supply chain system configures a typical hybrid push-pull system, in which product flow is partially operated by the schedule-driven method, and partially operated by the buffer-driven method. With this combination, the system should include various types of information flows, and the configuration of the flow is also very complex. This has been a good case for us to extract information of the supply chain operations. This supply chain scenario has been developed and will be the topic for another report.

Figure 1 represents the result of a configuration analysis of the target supply chain system. This configuration is based on the case study of a particular supply chain of machinery products. However, the definitions of business process activities, specification of information, and relationship of the information component are generic and are applicable to common definitions of communication data in a supply chain system.

Symbols that are used in Figure 1 include:

- Rectangular box: denoting a supply chain member

- Arrow with thin line: denoting the information flow

- Arrow with thick line: denoting the products flow

- Dashed box: denoting an external data input

- Star: denoting a supply chain member associated with a particular company

In Figure 1, there are seven major players:

$\begin{array}{ll}\text { - } & \text { Headquarters } \\ \text { - } & \text { Parts suppliers } \\ \text { - } & \text { Retailer } \\ \text { - } & \text { Distributor } \\ \text { - } & \text { Final assembly plant } \\ \text { - Transportation network }\end{array}$

The scope of this analysis is in the areas of production and operations management. It includes demand projection, resource capacity planning, production planning, inventory management, ordering management for production and transportation, and monitoring of operations.

Production and planning operations can be classified into three categories by way of the operations duration: long-range planning, medium-range planning, and short-range planning. The long-range planning normally addresses issues that impact the firm from between two to five years or more in the future. This type of plan involves three general areas of consideration: (1) nature of the product(s) or service(s), (2) output capacity, and (3) production facility location. The mediumrange planning and short-range planning deal with issues for three years to three months in duration and three months or less in duration, respectively. The three general areas of concern for mediumrange and short-range plans are as follows: (1) human and resource management - for mediumrange, (2) facility layout planning — for medium-range, and (3) production planning — for medium-range and short-range. 
The operations discussed in this case study belong to the short-range planning type. The planning in this study uses computer-based planning tools such as MRP (Material Requirement Planning), MPS (Master Production Schedule), CRP (Capacity Requirement Planning), and taps the knowledge of the manufacturing practitioners. This study uses a preliminary production plan with rough estimations provided by the manufacturers or supervisors. The study does not include product design operations and maintenance operations. Therefore, no product specification data is included in the data specification. 


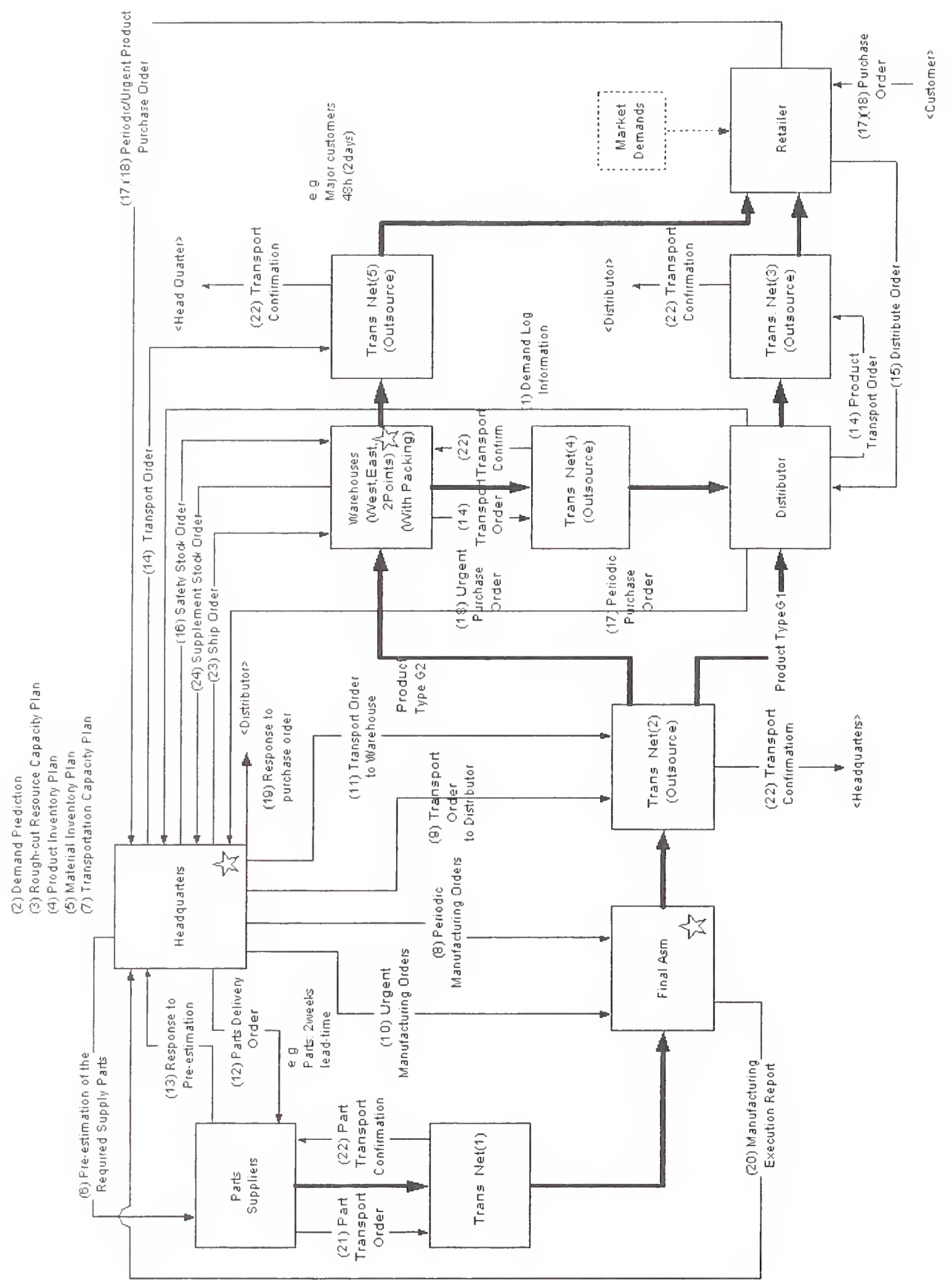

Figure 1. Information flow in a supply chain system 


\subsection{Requirements Analysis and Information Requirements}

A requirements analysis has been performed for the case study. We performed several interviews with a selected US manufacturing company, which plays a role of at prime contractor in their supply chain system. The operation flows and information flows in the supply chain were our focus. We identified a set of core processes of supply chain management and then analyzed the input and output messages within the core processes. As a result, the information required for the integration of the supply chain system was identified. These information requirements are a set of messitges, or objects, that are exchanged or shared among the supply chain members. Each of these messatges is defined by a set of attributes.

\subsection{Information Categories}

There are twenty-four major message types identified from the information flow analysis. These messages are listed as follows:

1) Demand log information

2) Demand prediction

3) Rough-cut resource capacity plan

4) Product Inventory plan

5) Material Inventory plan

6) Estimation of required supply parts

7) Transportation capacity plan

8) Periodic manufacturing order

9) Transport order to distributor

10) Urgent manufacturing order

11) Transport order to warehouse

12) Parts delivery order

13) Response to the Estimation

14) Transport order

15) Distribute order

16) Safety stock order

17) Periodic purchase order

18) Urgent purchase order

19) Response to purchase order

20) Manufacturing execution report

21) Part transport order

22) Transport confirmation

23) Shipment order

24) Supplement stock order

Each message/application object is an atomic element that embodies a unique applicattion concept and contanns attributes specifying the data elements of the object. The application objects and their definituons are listed below. 


\subsubsection{Demand log information}

The demand log information specifies the unprocessed (raw) data from the distribute orders, which the distributor receives from the retailer. This information is used to make a projection of the future demand.

The data associated with demand log information is the following:
Order ID
- the identifier of the order, given by the distributor.
Product ID
- the identifier of the product, specified by the distributor in the order.
Product Name - the name of the product, specified by the distributor in the order.
Product Quantity - the quantity of the product, specified by the distributor in the order.
Order Reception Date - the date, when the headquarters receives order from the distributor.
Distributor ID - the identifier of the distributor, who receives orders.
Distributor Name - the name of the distributor, who receives orders.
Retailer ID - the identifier of the retailer, who sends orders to the distributor.
Retailer Name - the name of the retailer that sends orders to the distributor.

\subsubsection{Demand prediction}

The demand prediction specifies the headquarters' projected volume of demand for the next business phase.

The data associated with demand prediction is the following:
Start Date
- the beginning date of the duration of the headquarters' projection.
End Date
- the ending date of the duration of the headquarters' projection.
Number of List Items - the record number of the products contained in the demand prediction.
List of Product ID, Product Name, Product Type, Product Quantity
- the list of product's identification, name, type, and predicated quantity, specified in the demand prediction.

\subsubsection{Rough-cut resource capacity plan}

The rough-cut resource capacity plan specifies the estimated utilization of the enterprise resources, such as human, machinery, and monetary resources, to perform the production. The resource estimation is based on the demand prediction. This resource estimate is expressed in terms of the production volume. The resource is converted to production volume during the specific term. For example, suppose that three machines and two operators are available in the next month, and $\$ 30,000$ is available to purchase parts for manufacturing. In this case, this factory would be able to produce 200 pieces of "product A" and 100 pieces of "product B" in the duration. Such conversion will be directly reflected in the production planning.

The data associated with rough-cut resource capacity plan is the following:

Start Date - the beginning date of the duration of planning phase, defined in the rough-cut resource capacity plan.

End Date - the ending date of the duration of planning phase, defined in the roughcut resource capacity plan.

Number of List Items - the record number of the product items, contained in the rough-cut resource plan.

List of Product ID, Product Name, Product Quantity 
- the list of product's identification, name, and predicated quantity, specified in the rough-cut capacity plan.

\subsubsection{Product Inventory plan}

The product inventory plan specifies the volume of the product inventory in the duration of planning. The volume of demand fluctuation and the rate of product inventory rotation have to follow the management policies.

The data associated with product inventory plan is the same as those with demand predication (refer to 3.1.2.)

\subsubsection{Material Inventory plan}

The material inventory plan specifies the volume of parts inventories in the duration of planning. This is based on the production inventory plan and part inventory rotation rate.

The data associated with material inventory plan is the following:

Start Date - the beginning date of the duration of planning phase, defined in the part inventory plan.

End Date - the ending date of the duration of planning phase, defined in the part inventory plan.

Number of List Items - the record number of the material items, contained in the material inventory plan.

List of Part ID, Part Name, Part Quantity, Inventory Location ID, Inventory Location Name

- the list of part's identification, name, quantity, inventory location identifier, and inventory location, specified in the material inventory plan.

\subsubsection{Estimation of required supply parts}

The estimation of required supply of parts specifies the estimated volume of the parts, which are consumed in the downstream process (e.g. the final assembly plant). This is not a formal order to the supplier, but a projection about the consumption of parts in the down-stream factories. The suppliers can use this data to predict the load factor in the next phase and to send a response. For example, if an over-capacity of parts volume is estimated, an "accepted" or "rejected" response will be sent, otherwise, a "partially" response will be sent. Estimation of required supply parts and response to the estimation are used to negotiate purchasing of parts.

The data associated with estimation of required volume of parts is the following:

Estimation ID

Start Date

End Date
- the identifier of the estimation of required volume of parts.

- the beginning date of the duration of estimation, which the headquarters gives to the suppliers with the estimation of required volume of parts.

- the ending date of the duration of estimation, which the headquarters gives to the suppliers with the estimation of required volume of parts.

Number of List Items - the record number of the material items, contained in the estimation of required volume of parts.

List of Part ID, Part Name, Part Quantity 
- the list of part's identification, name, and quantity, specified in the estimation of required volume of parts.

\subsubsection{Transportation capacity plan}

The transportation capacity plan specifies the requirements for transportation between the suppliers. The requirements are based on the demand during the planning phase.

The data associated with transportation capacity plan is the following:

Transportation ID - the identifier of the transporter, specified in the transportation capacity plan.

Transportation Name - the name of the transporter, specified in the transportation capacity plan.

From Location ID - the identifier of the upstream company or organization, specified in the transportation capacity plan.

From Location Name - the name of the upstream company or organization, specified in the transportation capacity plan.

To Location ID - the identifier of the downstream company or organization, specified in the transportation capacity plan.

To Location Name - the name of the downstream company or organization, specified in the transportation capacity plan.

Start Date - the start date of the duration of estimation, specified in the transportation capacity plan. End Date - the end date of the duration of estimation, specified in the transportation capacity plan.

Estimated Total Capacity

- the estimated total material weight, which the transporter can carry during the defined period.

\subsubsection{Periodic manufacturing order}

The periodic manufacturing order specifies the manufacturing order sent to the factory within the firm. The planner, such as the headquarters, gives the manufacturing order to the manufacturing line. The order volume is dependent on several manufacturing plans, including product inventory plan, safety stock volume, etc.

The data associated with periodic manufacturing order is the following:

Order ID

Order Date

Number of List Items - the record number of products, specified in the periodic manufacturing order.

List of Product ID, Product Name, Ordered Product Quantity, Due Date, Cost of Manufacturing - the list of product's identification, name, and order quantity, the due date for this product, and the cost of the volume of this product, specified in the periodic manufacturing order.

Ordering Cost - the total cost, including products fee and transportation fee, to perform the periodic manufacturing order.

\subsubsection{Transport order to distributor}


The transport order to distributor specifies the order for transporting products from the upstream organization to the distributor. The planner, such as the headquarters, gives the order to the transporter to deliver products to the distributor. The order is synchronized with the periodic mannfacturing order and the urgent manufacturing order. The data associated with transport order to distribntor is the same as those with transport order (refer to 3.1.14).

\subsubsection{Urgent manufacturing order}

The urgent mannfacturing order specifies a special manufacturing order sent to the factory within the firm. The planner gives the manufacturing order regularly, based on the master production plans, and from time to time the headquarters issues special orders. Urgent manufacturing order is a special order that is not included in the periodic orders.

The data associated with urgent mamufacturing order is the following:
Order ID
- the identifier of the urgent manufacturing order, which is given by the headquarters.
Order Date
- the order published date, specified in the urgent manufacturing order.
Product ID
- the identifier of the product, specified in the headquarters' nrgent manufacturing order.
Product Name - the name of the product, specificd in the headquarters' urgent manufacturing order.
Order Quantity
- the quantity of the product, specified in the headquarters' urgent
Due Date manufacturing order.
- the date, which the final assembly plant must finish the work for the urgent manufacturing order.
Distributor ID - the identifier of the distributor, which requested the urgent mannfacturing order.
Distributor Name - the name of the distributor, which requested the urgent mannfacturing order.
Retailer ID
- the identifier of the retailer, which requested the urgent manufacturing order.
Retailer Name - the name of the retailer, which requested the urgent manufacturing order.
Ordering Cost - the total cost to perform the urgent manufacturing order.

\subsubsection{Transport order to warehouse}

The transport order to warehonse specifies the order that is used to transport products from the upstream organization to the warehouse. The planner gives this order to the transporter to deliver the products to the warehouse. This order is synchronized with the periodic manufacturing order and the urgent manufacturing order. The data associated with transport order to distributor is the same as those with transport order (refer to 3.1.14).

\subsubsection{Parts delivery order}

The parts delivery order specifies the order sent to the part suppliers for purchasing the parts. The ordered volume and duration is based on the material inventory plan. 
The data associated with parts delivery order is the following:

Order ID

Order Date

Part ID

Part Name

Order Quantity

Due Datc

Part Supplier ID

Part Supplier Name

Desirable Price
- the identifier of the parts delivery order, which is given by the headquarters.

- the date, when the parts delivery order is published.

- the identifier of the part, specified in the headquarters' parts delivery order.

- the name of the part, specified in the headquarters' parts delivery order.

- the quantity of the part, specified in the headquarters' parts delivery order.

- the date, which the parts supplier plant must finish the work for the parts delivery order.

- the identifier of the supplier that receives the parts delivery order.

- the name of the supplier that receives the parts delivery order.

- the price, which the headquarters desires for the parts delivery order.

\subsubsection{Response to the estimation}

The response to the estimation specifies the supplier's response to the estimation of required volune of parts. This is a response to the projection about parts consumption in the down-stream factories.

The data associated with response to the estimation is the following:

Response ID

Estimation ID

Response

- the identifier of the response to the estimation.

- the identifier of the estination of required volume of parts.

- the supplier's response to the estimation of required volume of parts. If supplier can cope with an addition or cancellation, the response will be an "accepted." If the supplier cannot cope with an addition or cancellation, the response will be a "rejected." Otherwise, the supplier responds a "partially" to the planner. A "partially" response means that the given estimation is acceptable, if the volumes on some particular parts could be reduced.

Response Date - the date when the response to the estimation is published.

List of Part ID, Part Name, Part Quantity

- a new list of part's identification, name, and reduced volume of parts, which are estimated by the supplier.

\subsubsection{Transport order}

The transport order specifies the order sent to the internal or external transporter for delivering the products to the assigned destination.

The data associated with transport order is the following:

Order ID

Departure ID

Departure Name
- the identifier of the transport order.

- the identifier of the upstream supplier of the transporter, specified in the transport order.

- the name of the upstream supplier of the transporter, specified in the transport order. 
Destination ID - the identifier of the downstream supplier of the transporter, spccified in the transport order.

Destination Name - the name of the downstream supplier of the transporter, specified in the transport order.

Desirable Date - the desirable date, which the transportation of the transport order finishes.

Due Date - the date, which the transportation of the tratsport order must finish.

Duration - the minimum duration, which the transportation is performed.

Number of List Items - the record number of the products, specified in the transport order.

List of Product ID, Product Name, Product Type, Product Weight, Product Quantity

- the list of product's identification, name, type, weight, and quantily, specified in the transport order.

Total Wcight

- the products' total weight, which the transportation executes.

Total Price

- the total price of the products, which the transportation exccutes.

Shipping cost

- the expense, which this transportation charges.

\subsubsection{Distribute order}

The distribute order specifies the order used for shipping products to the retailers. The Distributor receives this order from the retailer.

The data associated with distribute order is the following:

Order ID

Retailer ID

Retailer Name

Distributor ID

Distributor Name

Desirable Date

Due Date

Duration

Number of list items

- the identifier of the distribute order.

- the identifier of the retailer that sends the distribute order.

- the name of the retailer that sends the distribute order.

- the identifier of the distributor that receives the distribute order.

- the name of the distributor that receives the distribute order.

- the date, which the retailer desires to get products from the distributor.

- the date, which the retailer must get products from the distributor.

- the minimum duration, which the distribute order is performed.

List of Product ID, Product Name, Product Type, Product Weight, Product Quantity

- the list of product's identification, name, type, weight, and quantity, specified in the distribute order.

Total Weight

Total Price

- the weight of the total of products, specified in the distribute order.

- the total price of the products, specified in the distribute order.

\subsubsection{Safety stock order}

The sufety stock order specifies the criteria for the quantity of the safety stock in the warchouse. When the product volume in the warehouse becomes less than the criteria, the upstrcam supplicr replenishes the products. The headquarters decides these criteria and informs individual warehouses in every planning phase.

The data associated with safety stock order is the following:

Warehouse ID - the identifier of the warehouse.

Warehouse Name - the name of the warehouse.

Number of List Items - the record number of the products, specified in the safery stock order. 
List of Product ID, Product Name, Safety Stock Quantity, Order Date

- the list of the product's identification, name, and safety stock quantity, and the order published date, specified in the safety stock order. The warehouse gives the safety stock order to the headquarters, when the current holding volume of products becomes below the specified safety stock quantity. The safety stock quantity or stock criteria is determined by the headquarters.

\subsubsection{Periodic purchase order}

The periodic purchase order specifies the order, with which the distributors or the retailers purchase the products. The order is sent to the headquarters in regular cyclic duration, (e.g. every Monday morning.) The order volume is based on the requests from the customers, and the distributors' or retailers' product inventory policies.

The data associated with periodic purchase order is the following:

Purchase Order ID - the identifier of the periodic purchase order sent from the retailer or distributor.

Purchase Order Date - the date when the periodic purchase order is published.

Number of List Items - the record number of the products, specified in the periodic purchase order.

List of Product ID, Product Name, Product Type, Product Quantity, Price

- the list of product's identification, name, type, quantity, and price, specified in the periodic purchase order.

Due Date - the date, when the retailer or distributor must to receive the products from their upstream.

Retailer ID - the identifier of the retailer that sent the periodic purchase order.

Retailer Name - the name of the retailer that sent the periodic purchase order.

Distributor ID - the identifier of the distributor that sent the periodic purchase order.

Distributor Name - the name of the distributor that sent the periodic purchase order.

Total Price - the price to perform the purchase.

\subsubsection{Urgent purchase order}

The urgent purchase order specifies the order, with which the distributors or the retailers purchase the products from this company. The order is sent to the headquarters only when the distributor or the retailer needs additional orders. The order volume is based on the requests from their customers and their product inventory policies.

The data associated with urgent purchase order is the following:

Purchase Order ID - the identifier of the urgent purchase order sent from the retailer or distributor.

Purchase Order Date - the date when the urgent periodic purchase order is published.

Product ID

- the identifier of the product, specified in the urgent purchase order.

Product Name

- the name of the product, specified in the urgent purchase order.

Order Quantity

Due Date

- the quantity of the product, specified in the urgent purchase order.

- the date, when the retailer or distributor must to receive the products from their upstream. 
Retailer ID

Retailer Name

Distributor ID

Distributor Name

Total Price
- the identifier of the retailer that sent the urgent purchase order.

- the name of the retailer that sent the argent purchase order.

- the identifier of the distributor that sent the urgent purchase order.

- the name of the distributor that sent the urgent purchase ordet.

- the price to perform the purchase.

\subsubsection{Response to purchase order}

The response to purchase order specifies the information sent to respond to the periodic or urgent purchase order. The response indicates whether the order can be filled.

The data associated with response to purchase order is the following:

Purchase Response ID - the identifier of the response to purchase order.

Purchase Order ID - the identifier of the purchase order sent by the retailer or distributor.

Number of List Items - the record number of the products, specified in the response to purchase order.

List of Product ID, Product Name, Product Quantity, Price

- the list of product's identification, name, type, quantity, and price, specified in the response to purchase order.

Due Date - the date, when the retailer or distributor requires to get products from their upstream organization.

Planned Delivery Date - the date, when the delivery is available.

Distributor ID - the identifier of the distributor that sends the periodichurgent purchase order.

Distributor Name - the name of the distributor that sends the periodic/urgent purchase order.

Retailer ID - the identifier of the retailer that sends the periodichurgent purchase order.

Retailer Name - the name of the retailer that sends the periodichurgent purchase order.

Total Price

Order Class

- the price, which the purchase is performed.

- the class of the purchase order. Order class can be the class of " $R$ " for a regular purchase or the class of "U" for an urgent purchase.

\subsubsection{Manufacturing execution report}

The mamufacturing execution report specifies the execution report about the factory. The report identifies the factory's throughputs of the products.

The data associated with manufacturing execution report is the following:

Report Sequence ID - the identifier of the manufacturing execution report.

Execution Report Date

- the date when the manufacturing execution report is published.

Number of List Items - the record number of the products, specified in the manufacturing execution report.

List of Product ID, Product Name, Product Type, Product Quantity

- the list of product's identification, name, type, and quantity, specified in the manufacturing execution report. 


\subsubsection{Part transport order}

The part transport order specifies the transport order, with which the transportation network carries the parts of product from the parts suppliers to the product factory.

The data associated with part transport order is the following:
Order ID
- the identifier of the part transport order.
From Location ID - the identifier of the upstream facility of the transportation.
From Location Name - the name of the upstream facility of the transportation.
To Location ID - the identifier of the downstream facility of the transportation.
To Location Name - the name of the downstream facility of the transportation.
Desirable Date - the date when the retailer or distributor desires to get the products from their upstream.
Due Date - the last date when the downstream must get products from their upstream.
Duration - the time required to accomplish the transportation.
Number of List Items - the record number of the products, specified in the part transport.
List of Product ID, Product Name, Product Type, Product Weight, Product Quantity
- the list of product's identification, name, type, weight, and quantity, specified in the part transport order.
Total Weight - the total weight of the products in this part transportation.
Total Shipping Cost - the total shipping cost required for this transportation.

\subsubsection{Transport confirmation}

The transport confirmation specifies the performance status about the transportation between suppliers.

The data associated with transport confirmation is the following:

Transport Order ID - the identifier of the part transport order.

Confirmation Code - the status identifier about the performance of the part transport order. The status may be "on time," "delayed," or "cancelled."

Days of Delay - is the number of days of delay, when the part transport order is delayed.

Reason - the reason why the part transport order is delayed or cancelled.

\subsubsection{Shipment order}

The shipment order specifies the shipment order used to ship the products to the retailer.

The data associated with shipment order is the following:

Ship Order ID - the identifier of the shipment order.

Number of List Items - the record number of the products, specified in the shipment order.

List of Product ID, Product Name, Product Type, Product Quantity

- the list of product's identification, name, type, and quantity, specified in the shipment order.

Warehouse ID - the identifier of the warehouse from which the products are shipped to the customer. 
Warehouse Name - the name of the warehouse from which the products are shipped to the customer.

Desirable Date - the desirable date when the products arrive at the destination.

Due Date - the latest date when the products must arrive at the destination.

Destination Type - the identifier of the type of the destination. Destination may be at the distributor or the retailer location.

Destination ID - the identifier of the destination of the shipment.

Destination Name - the name of the destination of the shipment.

\subsubsection{Supplement stock order}

The supplement stock order specifies the order, requested by a warehouse to fulfill the replenishment point of products. A warehouse holds the minimum inventory of product (replenishment point) in order to respond to the shipment requests.

The data associated with supplement stock order is the following:

Supplement Stock Order ID

- the identifier of the supplement stock order.

Number of List Items - the record number of the products, specified in the supplement stock order.

List of Product ID, Product Name, Product Type, Product Quantity

- the list of product's identification, name, type, and quantity, specified in the supplement stock order.

Order Date - the date when this order is placed.

Warehouse ID - the identifier of the warehouse placing the order.

Warehouse Name - the name of the warehouse placing the order.

\subsection{Information Exchange Specification}

This section presents a data specification that describes, with a formal syntax, the information requirements listed in Section 3.1. The specification is used for archiving, sharing, and exchanging the supply chain messages within the scenario described in the previous sections. The data specification is presented using the eXtensible Markup Language (XML) language definition, or XML data type definitions (XML DTD) [6]. Subsection 4.1 gives an overview of the XML language. The expression of the proposed data specification using the XML DTD is presented in the subsection 4.2. Appendix $\mathrm{A}$ is a sample XML document containing physical data.

\subsection{Introduction to XML}

$\mathrm{XML}$ is a neutral format for structuring documents and data on the Web. The language helps make information exchange among a globally distributed computing environment possible. XML and its sibling HTML (Hyper Text Markup Language) are based on the Standard Generalized Markup Language (SGML) [7], the international standard for structured information. XML is a simplified subset of SGML. The simplification is optimized for the Web environment, which implies dataprocessing-oriented, short-term information. While HTML defines the output format on WEB pages, XML defines semantics of the information and structures of the documents.

XML, by virtue of user defined tags, allows precise encoding of structured information. Structured information contains both data and an indication of the meaning of the data. The markup in XML 
defines the meaning of the data. The role of the markup is therefore different from that in HTML. In HTML the markup defines a view of the data, i.e., it instructs the browser how to display the data. XML relies on application programs or style sheets to manipulate, edit, and present the data. This separation of the data from the presentation and the process allows for applications that can be built more quickly, are easier to maintain, and can provide multiple views of data from any source. XML is valuable to the Web because it provides interoperability using a flexible, open, standardsbased format for structured information. It will enable a new generation of Web-based applications for viewing, searching, and manipulating data.

XML is a text-based format. It is for the digital representation of documents. An XML document is defined as a series of characters. XML provides a mechanism for allowing text to be organized non-linearly and potentially in multiple pieces.

The following example is an XML document containing a document type declaration and document type definition (DTD) for mailing addresses, followed by an instance of the document type, a single address. A DTD is a series of definitions for element types, attributes, entities, and notations. A document can claim to conform to a particular DTD in its document type declaration.

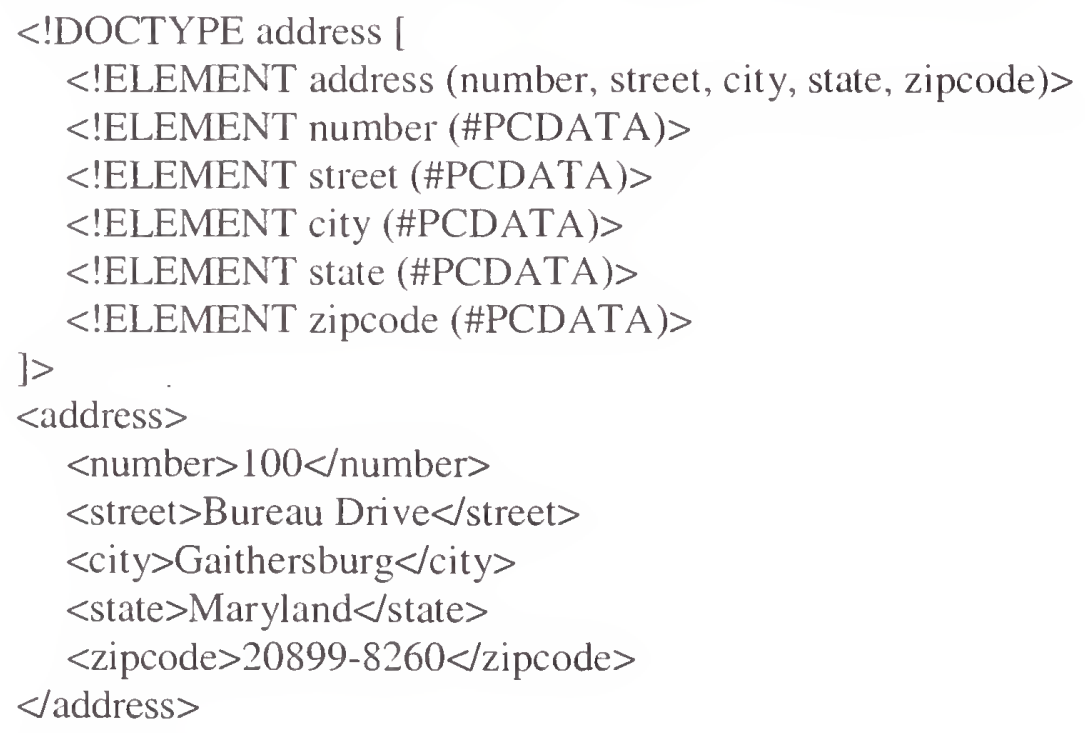

The example above defines an element named "address" that contains 5 elements: address, number, street, city, state, and zip code. The document type declaration starts with " $<$ !DOCTYPE" and ends with "I>". The element type declarations are lines starting with "<!ELEMENT". The DTD declares the tags and their contents. In the above example, all of the DTD declarations that define the address DTD reside within the document entity. The DTD could have been defined partially or completely somewhere else. In that case, the document type declaration would contain a reference to another entity containing those declarations. As the document type declaration is optional, a well-formed XML document can choose not to declare conformance to any DTD. It can not then be a valid document because it cannot be tested for conformance to a DTD. 


\subsection{XML Specification}

In this subsection, the Supply Chain Data Specification is presented in the XML DTD. XML is case-sensitive. An XML source is made up of XML elements, each of which consists of a start tag $<$ element name>, an end-tag </element name>, and the information between the two tags (referred to as the content). An element can contain other elements and attributes. Attributes are name-value pairs that present inside start-tags after the element name. The respective value is enclosed by quotes, e.g., Year $=$ "2000". Comments begin with $<$ !-- and end with -->. The following is the XML specification.

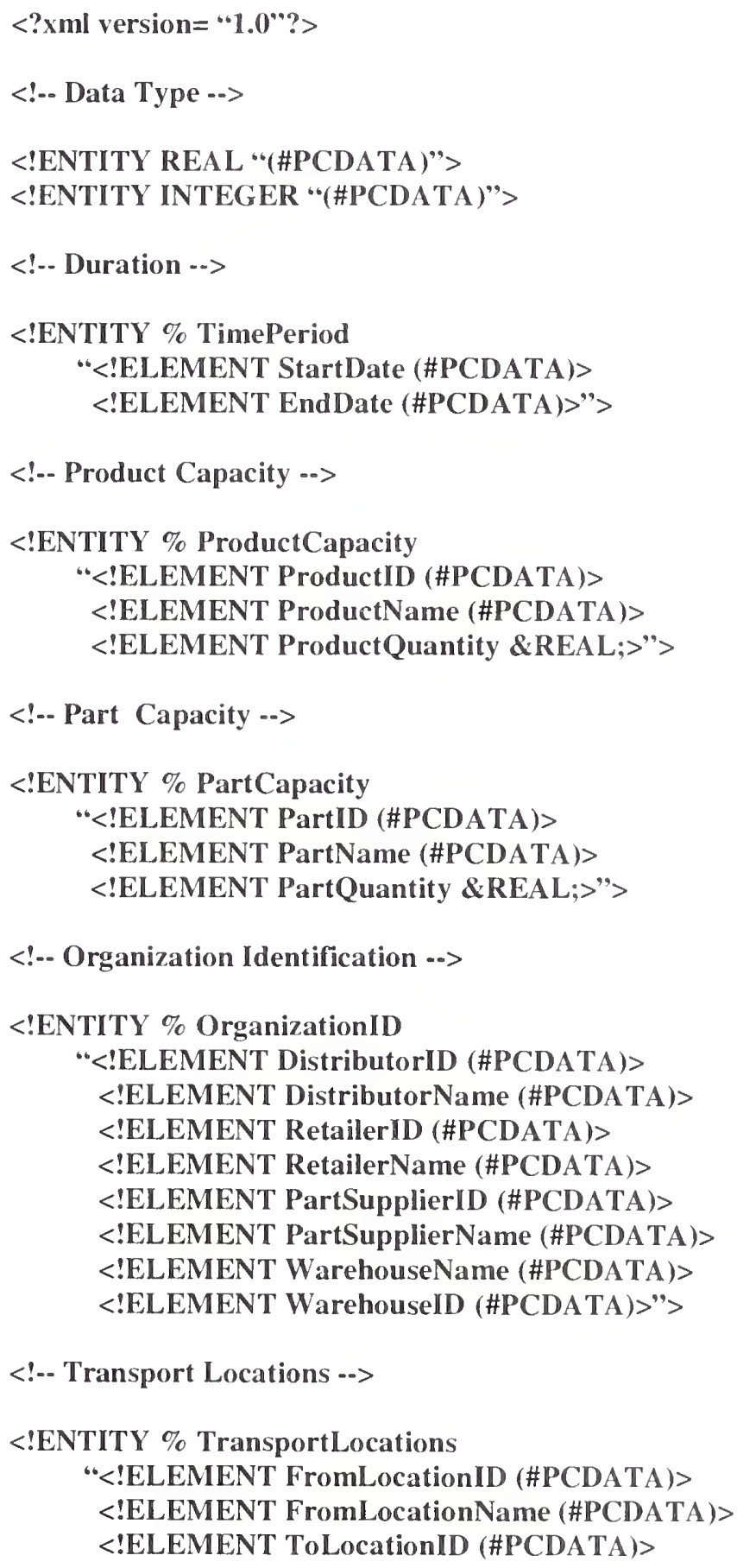


$<$-- Transport Total -.>

$<$ :ENTITY \% TransportTotal

"<!ELEMENT TotalWeight \&REAL; $>$

$<$ !ELEMENT TotalPrice \&REAL;>">

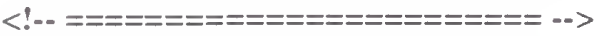

$<$-- Entity References $\quad$-->

$<!--=======================~-->~$

\% TimePeriod;

\% ProductCapacity;

\% PartCapacity;

\%OrganizationII);

\% TransportLocations;

\% TransportTotal;

$<$ !-- SupplyChain -->

$<$ !ELEMENT SupplyChain (Product*, Denand LogInfo*, DemandPrediction?,

ResourceCapacityPlan?, ProductInventoryPlan?, MaterialInventoryPlan?,

PartsRequirement?, TransporationPlan*, PeriodicOrder?,

OrderToDistributor*, UrgentOrder*, OrderToWarehouse*,

PartsDeliveryOrder*, EstimationResponse*, TransportOrder*,

DistributeOrder*, SafetyStockOrder*, PeriodicPurchaseOrder*, UrgentPurchaseOrder*, PurchaseOrderResponse?, ManufacturingExecutionReport?, PartTransportOrder*, TransportConfirmation*,

ShipnentOrder*, SupplementStockOrder*)>

$<!--$ Product -->

$<$ :ELEMENT Product (ProductID, ProductName?, ProductPrice?, ProductQuantity?,

Product Weight?)>

$<$ !ELEMENT ProductPrice \&REAL;>

$<$ !ELEMENT ProductWeight \&REAL; $>$

$<!-$ Distributor or Retailer -.>

$<$ !ELEMENT Distributor (DistributorID, DistributorName?) $>$

$<$ 'ELEMENT Retailer (RetailerID, RetailerName?) >

$<$ 'ELEMENT DistributorOrRetailer (Distributor | Retailer) >

$<$ !-- Demand Log Information -->

$<$ !ELEMENT Demand LogInfo (OrderID, ProductID, ProductName?, ProductQuantity, OrderReceptionDate?, DistributorOrRetailer)>

$<$ ?ELEMENT OrderID (\#PCDATA)>

$<$ ?ELEMENT OrderReceptionDate (\#PCDATA) >

$<!-$ Product Item -- >

$<$ !ELEMENT ProductItem (ProductID, ProductName:, ProductType?, ProductQuantity) >

$<$ !-- Demand Prediction --> 
$<$ !ELEMENT DemandPrediction (StartDate, EndDate, NumberOf ListItems, ProductItem+) > $<$ !ELEMENT NumberOfListltens \& INTEGER; >

$<!--$ Rough-cut Resource Capacity Plan -->

$<$ !ELEMENT ResourceCapacityPlan (StartDate, EndDate, NumberOfListltems, I'roductl tem+) >

$<$ !-- Product Inventory Plan -->

$<$ !ELEMENT ProductInventoryPlan (StartDate, EndDate, NumberOfListItems, ProductItem+)>

$<!-$ Part Inventory Item -->

$<$ 'LLEMENT PartlnventoryItem (PartID, PartName?, PartQuantity, Inventory LocationlI),

Inventory Location?)>

$<$ :ELEMENT InventoryLocationID (\#PCDATA) >

$<$ !ELEMENT InventoryLocation (\#PCDATA) >

$<!--$ Material Inventory Plan -->

<!ELEMENT MaterialInventoryPlan (StartDate, EndDate, NumberOfListItems, PartInventoryltem+) >

$<!-$ Part IItem -->

$<$ !ELEMLNT Partltem (PartII, PartName?, PartQuantity)>

$<$-- Estimation of required Quantity of parts -->

$<$ !ELEMENT PartsRequirement (EstimationID, StartDate, EndDate, NumberOfListI tems,

PartItem+)>

$<$ !ELEMENT EstimationID (\#PCDATA)>

$<$ !-- Transporation Capacity Plan -->

$<$ !ELEMENT TransporationPlan (TransportationID, TransportationName?, FromLocationID,

FromLocationName?, ToLocationIID, ToLocationName?, StartDate, End Date,

EstimatedTotalCapacity)>

$<$ !ELEMENT TransportationID (\#PCDATA) >

$<$ !ELEMENT TransportationName (\#PCDATA) >

$<$ !ELEMENT EstimatedTotalCapacity \&REAL;>

$<!-O r d e r e d$ Product Item -->

$<$ :ELEMENT OrderedProductI tem (ProductID, ProductName?, ProductQuantity', DueDate,

CostOfManufacturing)>

$<$ !ELEMENT DueDate (\#PCDATA)>

$<$ !ELEMENT CostOfManufacturing \& REAL; >

$<$ !-- Periodic Manufacturing Order -->

$<$ !ELEMENT PeriodicOrder (OrderID, OrderDate, Number OfListI tems, OrderedProduct Item+, OrderingCost)>

$<$ !ELEMENT OrderDate (\#PCDATA) >

$<$ !ELEMENT OrderingCost \&REAL;> 
$<$ !-- Urgent Manufacturing Order -.>

$<$ :ELEMENT UrgentOrder (OrderID, OrderDate, ProductID, ProductName?, OrderQuantity, DueDate, DistributorOrRetailer, OrderingCost)>

$<$ :ELEMENT OrderQuantity \&REAL;>

$<$ !ELEMENT DistributorOrRetailerID (\#PCDATA) >

$<$ 'ELEMENT DistributorOrRetailerName (\#PCDATA) >

$<$ !-- Parts Delivery Order -.>

$<$ :ELEMENT PartsDeliveryOrder (OrderID, OrderDate, PartID, PartName?, OrderQuantity, DueDate, PartSupplierID, PartSupplierName?, DesirablePrice)>

$<$ !ELEMENT DesirablePrice \& REAL;>

$<$ !-- Response To the Estimation -- >

$<$ !ELEMENT EstimationResponse (ResponseID, EstimationID, Response, ResponseDate,

Partially ResponseQuantity)>

$<$ !ELEMENT ResponseID (\#PCDATA)>

$<$ !ELEMENT Response >

$<$ :ATTLIST Response Code (Accepted | Rejected | Partially) \#REQUIRED>

$<$ !ELEMENT PartiallyResponseDate (\#PCDATA) >

$<$ :ELEMENT Partially ResponseQuantity \& REAL;>

$<$ !-- Transport Item -->

$<$ :ELEMENT TransportItem (ProductIID, ProductName?, ProductType?, ProductWeight?,

ProductQuantity)>

$<$ ?ELEMENT ProductType (\#PCDATA) >

$<$ !-- Transport Order -.>

$<$ !ELEMENT TransportOrder (OrderID, DepartureII), DepartureName?, DestinationID,

DestinationName?, DesirableDate?, DueDate, Duration, NoOfListltems, Transportltem+, TotalWeight,

TotalPrice, ShippingCost)>

$<$ 'ELEMENT DepartureID (\#PCDATA) >

$<$ !ELEMENT DepartureName (\#PCDATA)>

$<$ !ELEMENT DestinationID (\#PCDATA) >

$<$ :ELEMENT DestinationName (\#PCDATA) >

$<$ !ELEMENT DesirableDate (\#PCDATA) >

$<$ !ELEMENT Duration (\#PCDATA) >

$<$ 'ELEMENT ShippingCost \& REAL;>

$<$ !-- Transport Order to Distributor-->

$<$ !ELEMENT OrderToDistributor (OrderI D, DepartureID, DepartureName?, DestinationII), DestinationName?, DesirableDate?, DueDate, Duration, NoOfListItems, Transportltem+, TotalWeight, Totall'rice, ShippingCost)>

<!-- Transport Order to Warehouse -->

$<$ !ELEMENT OrderToWarehouse (OrderID, DepartureID, DepartureName?, DestinationID, IestinationName?, DesirableDate?, DueDate, Duration, NoOfListltems, Transportltem+, TotalWeight, TotalPrice, ShippingCost)>

$<$ !-- Distribute Item -.> 
$<$ !ELEMENT Distributeltem (ProductID, ProductName?, ProductType?, ProductWeight?, ProductQuantity)>

$<!--$ Distribute Order -->

<!ELEMENT DistributeOrder (OrderID, RetailerID, RetailerName?, DistributorID, DistributorName?, DesirableDate, DueDate, Duration, NoOfListItems, Distributeltenıt, TotalWeight, TotalPrice)>

$<$ !-- Safety Stock Order Item -->

$<$ :ELEMENT SafetyStockOrderItem (ProductID, ProductName?, SafetyStockQuantity, OrderDate) > $<$ :ELEMENT SafetyStockQuantity (\#PCDATA)>

$<$ ?-- Safety Stock Order On Criteria -->

$<$ :ELEMENT SafetyStockOrder (WarehouseID, WarehouseName?, NoOfListItems, SafetyStockOrderItem+)>

$<!--$ Purchase Item -- >

$<$ !ELEMENT PurchaseItem (ProductID, ProductName?, ProductType?, ProductQuantity, Price) $>$ $<$ !ELEMENT Price \&REAL;>

$<!-$ Periodic Purchase Order -->

$<$ :Element PeriodicPurchaseOrder (PurchaseOrderID, PurchaseOrderDate, NumberOflistItems, PurchaseItem+, DueDate, DistributorOrRetailer, TotalPrice) $>$

$<$ !ELEMENT PurchaseOrderID (\#PCDATA) >

$<$ !ELEMENT PurchaseOrderDate (\#PCDATA) >

$<$ !-Urgent Purchase Order -->

$<$ :ELEMENT UrgentPurchaseOrder (PurchaseOrderID, PurchaseOrderDate, ProductID, ProductName?, ProductQuantity, DueDate, DistributorOrRetailer, TotalPrice)>

$<$-- Response To Purchase Order -->

$<$ !ELEMENT PurchaseOrderResponse (PurchaseResponseID, PurchaseOrderID, NumberOfListItems, PurchaseItem+, DueDate, PlannedDelivery Date, DistributorOrRetailer, TotalPrice, OrderClass)> $<$ !ELEMENT PurchaseResponseID (\#PCDATA)>

$<$ !ELEMENT PlannedDeliveryDate (\#PCDATA)>

$<$ !-- Manufacturing Execution Report -->

$<$ !ELEMENT ManufacturingExecutionReport (ReportSequenceII, ExecutionReportDate, NumberOfList Items, ProductItem+)> $<$ !ELEMENT ReportSequenceID (\#PCDATA) > $<$ :ELEMENT ExecutionReportDate (\#PCDATA) >

$<$ !-- Part Transport Order $\ldots>$ 
$<$ !ELEMENT PartTransportOrder (OrderID, FromLocationID, FromLocationName?, ToLocationID, ToLocationName?, DesirablelDate, DueDate, Duration, NoOfListltems, Transportlltem+, TotalWeight, ShippingCost)>

$<$ !-- Transport Confirmation-->

$<$ :ELEMENT TransportConfirmation (TransportOrderII), Confirmation, I)aysOfDelay?, Reason?) > $<$ !ELEMENT TransportOrderID (\#PCDATA) >

$<$ !ELEMENT DaysOfDelay \&INTEGER;>

$<$ !ELEMENT Reason (\#PCDATA) >

$<$ !ELEMENT Confirmation>

$<$ :ATTLIST Confirmation Code ( OnTime | Delayed | Cancelled ) \#REQUIRED>

$<!--$ Shipment Order-->

$<$ !ELEMENT ShipmentOrder (ShipOrderID, NumberOfListItems, Productl tem+, WarehouseII), WarehouseName?, DesirableDate, DueDate, DestinationTy pe?, DestinationIID, DestinationName?)> $<$ !ELEMENT ShipOrderID (\#PCDATA) >

$<$ !ELEMENT DestinationType $>$

$<$ :ATTLIST DestinationType Code ( Distributor | Retailer) \#REQUIRED>

$<$ !-- Supplement Stock Order -->

$<$ 'ELEMENT SupplementStockOrder (SupplementStockOrderII), NumberOfListI tems, ProductItem+, OrderDate, WarehouselD, WarehouseName?)>

$<$ 'ELEMENT SupplementStockOrderID (\#PCDATA)> 


\subsection{Measurements Units}

The use of a particular system of measurement units is company-specific. However, use of mixed units will complicate the exchange and storage of data. Therefore, the units of measurement values used in this specification are predefined. We use "day" for the unit of time (duration), "dollar" for the unit of cost or price, "kg" for the unit of weight, and "number" for the unit of quantity.

\subsection{Conclusion}

A supply chain system is a group of companies that cooperate with each other to provide manufactured products to the market. Since these companies are independent, the contract(s) made between companies should be clearly defined. Also, because each chain company's requirements may and often do conflict with the others', and because some of the company's information and data may be confidential, developing a quality communication mechanism is required to realize a successful collaboration of chain member companies.

This report presents a data specification that provides a framework of communication data in a supply chain system. The data specification described in this paper can be used for the following purposes:

(1) Design of interfaces among the application programs to support supply chain operations: A supply chain system uses many application programs. The application developers must define input/output interfaces when designing such programs. The proposed specification will be contributions to define such interfaces for the individual applications.

(2) Data specification for Electronic Data Interchange (EDI): Electronic Commerce is a business trade using electronic communication. Electronic transfer of data is used instead of traditional paper-based contracts. A robust neutral data format is an enabling technology that facilitates the EDI implementation. The proposed data definitions using XML notation is a candidate for such a requirement.

(3) Specification of business communication: The successful implementation of a supply chain system depends on the realization of both a distributed decision support system and a welldefined communication mechanism. The proposed specification will be one of the fundamentals for development of these system enablers.

\section{Acknowledgments}

The authors thank Charles McLean and Swee Leong of NIST, and Mike Weaver of Black \& Decker for their technical assistance.

\subsection{References}

[1] MISSION Consortium, "Intelligent Manufacturing System (IMS) Project Proposal: Modelling and Simulation Environments for Design, Planning and Operation of Globally Distributed Enterprises (MISSION), Version 3.3," Shimuzu Corporation, Tokyo, Japan, March 1998.

[2] McLean Charles, Riddick, Frank, and Jones, Al, "An Architecture for Distributed Manufacturing Simulation," (To be published as a NISTIR), NIST, Gaithersburg, MD. 
[3] Simchi-Levi, David, Kaminsky, Philip, and Simchi-Levi, Edith, "Designing and Managing the Supply Chain: Concepts, Strategies, and Case Studies," Irwin McGraw-Hill, 2000.

[4] National Institute of Standard and Technology, "Electronic Data Interchange," Federal Information Processing Standards Publication 161-2, NIST, U.S., April 29, 1996.

[5] Lee, Y. T., "Information Modeling: From Design to Implementation," Proceedings of the Second World Manufacturing Congress, Durham, U.K., September 27-30, 1999.

[6] World Wide Web Consortium, "Extensible Markup Language (XML) 1.0," http://www.w3.org/TR/REC-xml.

[7] ISO/IEC JTC1/SC34/WG1, "ISO 8879: The Standard Generalized Markup Language (SGML)," http://www.ornl.gov/ggml;/wg4/.. 


\section{Appendix A: An Example XML Document}

$<!--$ This is an example XML document that contains 2 messages. -->

$<$ ? $\mathrm{xml}$ version $=" 1.0 " ?>$

$<!--$ An external DTD declarations: -->

<!DOCTYPE SUPPLYCHAIN SYSTEM "SupplyChain.dtd">

$<$ SupplyChain $>$

$<!--$ The document instances -- $>$

$<!$-DemandLogInfor is a message sent from a distributor to the headquarters. -->

$<$ !-- The message describes a projection of the future demand. -- $>$

$<$ DemandLogInfo $>$

$<$ OrderID $>$ DLI $1000</$ OrderID $>$

$<$ ProductID $>$ MS001 $<$ ProductiD $>$

$<$ ProductName $>$ Miter Saw $<$ ProductName $>$

$<$ ProductQuantity $>45000<$ ProductQuantity $>$

$<$ OrderReceptionDate $>30 J U N E 200 C</$ OrderReceptionDate $>$

$<$ DistributorID $>$ East $123<$ DistributorID $>$

$<$ DistributorName $>$ Baltimore Maryland $<$ DistributorName $>$

$<$ RetailerID $>$ HD $101<$ RetailerID $>$

$<$ RetailerName $>$ Home Depot Gaithersburg $<$ /RetailerName $>$

$<$ DemandLogInfo $>$

$<!-$ PeriodicPurchaseOrder is a message sent from a distributor or a retailer to the headquarters. -$>$

$<$ !-- The message is for purchasing product(s) periodically. -->

$<$ PeriodicPurchaseOrder $>$

$<$ PurchaseOrderID $>$ PO050100A $<$ PurchaseOrderID $>$

$<$ PurchaseOrderDate $>30$ AUGUST2000 $<$ PurchaseOrderDate $>$

$<$ NumberOfListItems $>1<$ NumberOfListItems $>$

$<$ PurchaseItem $>$

$<$ ProductID $>$ MS001 $<$ ProductID $>$

$<$ ProductName $>$ Miter Saw $</$ ProductName $>$

$<$ Product Type $>$ MS $<$ ProductType $>$

$<$ ProductQuantity $>3000<$ ProductQuantity $>$

$<$ Price $>10.50<$ Price $>$

$<$ Purchaseltem $>$

$<$ DueDate $>30$ September2000</DesirableDate $>$

$<$ DistributorID $>$ East $123<$ DistributorID $>$

$<$ DistributorName $>$ Baltimore Maryland $<$ DistributorName $>$ 
$<$ TotalPrice $>31500.00<$ TotalPrice $>$ $<$ PeriodicPurchaseOrder $>$

$<$ SupplyChain $>$ 

